\title{
High-Resolution In Vivo Imaging of the Neurovascular Unit during Spreading Depression
}

\author{
Julien Chuquet, Liad Hollender, and Esther A. Nimchinsky \\ Center for Molecular and Behavioral Neuroscience, Rutgers University, Newark, New Jersey 07102
}

Spreading depression (SD) is a propagating wave of neuronal depolarization and ionic shifts, seen in stroke and migraine. In vitro, SD is associated with astrocytic $\left[\mathrm{Ca}^{2+}\right]$ waves, but it is unclear what role they play and whether they influence cerebral blood flow, which is altered in SD. Here we show that SD in vivo is associated with $\left[\mathrm{Ca}^{2+}\right]$ waves in astrocytes and neurons and with constriction of intracortical arterioles severe enough to result in arrest of capillary perfusion. The vasoconstriction is correlated with fast astrocytic $\left[\mathrm{Ca}^{2+}\right]$ waves and is inhibited when they are reduced. $\left[\mathrm{Ca}^{2+}\right]$ waves appear in neurons before astrocytes, and inhibition of astrocytic $\left[\mathrm{Ca}^{2+}\right]$ waves does not depress SD propagation. This suggests that astrocytes do not drive SD propagation but are responsible for the hemodynamic failure seen deep in the cortex. Similar waves occur in anoxic depolarizations (AD), supporting the notion that SD and AD are related processes.

Key words: calcium waves; astrocytes; vasoconstriction; capillary flux; two-photon microscopy; cerebral ischemia

\section{Introduction}

Spreading depression (SD) is a response of the CNS to certain noxious stimuli. Originally described by Leão (1944), SD is a nondecremental wave, spreading at $2-5 \mathrm{~mm} / \mathrm{min}$, characterized locally by a suppression of electrical activity and a large negative direct current (DC) shift associated with redistribution of ions between extracellular and intracellular compartments (Somjen, 2001). It is believed to occur in several neurological disorders, including migraine (James et al., 2001), trauma (Strong et al., 2002; Fabricius et al., 2006), and stroke (Strong et al., 2002; Fabricius et al., 2006), in which it contributes to the death of compromised tissue (Busch et al., 1996; Nedergaard, 1996). Blockade of SD is an essential goal for the management of these disorders. So far, however, our understanding of its mechanism has proved inadequate for a successful therapeutic approach. Several cell populations participate in the initiation, propagation, and sequelae of SD, including neurons, glia, and vascular cells. Most studies of SD have been performed in vivo, but without cellular resolution, or in vitro and do not fully represent the situation in vivo, particularly with respect to hemodynamic changes.

SD was initially considered a neuronal phenomenon (Leão, 1944). However, the involvement of astrocytes was suggested by the discovery that, in vitro, astrocytes respond to $\mathrm{K}^{+}$, glutamate (Finkbeiner, 1992), or electrical stimuli (Dani et al., 1992), with $\left[\mathrm{Ca}^{2+}\right]$ waves propagating at a rate reminiscent of SD (MartinsFerreira et al., 2000; Haydon and Carmignoto, 2006). In vitro models of SD showed $\left[\mathrm{Ca}^{2+}\right]$ waves in astrocytes (Basarsky et al.,

Received Nov. 15, 2006; revised March 9, 2007; accepted March 10, 2007.

This work was supported by the National Science Foundation under Grant DBI-0320964. We thank Dr. Karim Benchenane for his participation in the early phase of this study and Drs. Gyorgi Buzsaki, Hajime Hirase, Brian A. MacVicar, and Patrick R. Hof for helpful comments.

Correspondence should be addressed to Dr. Esther A. Nimchinsky, Center for Molecular and Behavioral Neuroscience, Rutgers University, 197 University Avenue, Newark, NJ 07102. E-mail: nimchins@andromeda.rutgers.edu. DOl:10.1523/JNEUROSCI.0721-07.2007

Copyright $\odot 2007$ Society for Neuroscience $\quad$ 0270-6474/07/274036-09\$15.00/0
1998; Kunkler and Kraig, 1998; Peters et al., 2003), whose status as initiators of the wave or followers of neuronal processes remains unclear (Martins-Ferreira et al., 2000). Recently, astrocytic calcium has been linked to motion of vessels (Zonta et al., 2003; Filosa et al., 2004; Mulligan and MacVicar, 2004; Metea and Newman, 2006); the astrocytic wave of SD could therefore affect blood flow (Mulligan and MacVicar, 2004).

Several laser Doppler flowmetry (LDF) studies observed that, occasionally in rats (Fabricius et al., 1995; Dreier et al., 2001; Ayata et al., 2004; Tomita et al., 2005) and mice (Ayata et al., 2004), a brief hypoperfusion precedes the large transient hyperemia in SD. This can become a deleterious ischemia when nitric oxide is blocked or extracellular $\left[\mathrm{K}^{+}\right]$is artificially elevated (Dreier et al., 2001). This initial oligemia occurs during periinfarct depolarizations (Shin et al., 2006) and could explain the negative impact of SD on the ischemic penumbra. Thus, it is crucial to understand the mechanism of decreased blood flow during early SD (Fabricius et al., 1995).

Using an original approach, we demonstrate that SD dramatically affects blood flow deep in the intact cortex. We describe two astrocytic $\left[\mathrm{Ca}^{2+}\right]$ waves, one of which is associated with hemodynamic change. We show that a neuronal $\left[\mathrm{Ca}^{2+}\right]$ wave of SD generally precedes the astrocytic $\left[\mathrm{Ca}^{2+}\right]$ wave and that interference with the latter prevents vasoconstriction but not propagation of SD. Finally, we show that cardiac arrest-induced AD is accompanied by $\left[\mathrm{Ca}^{2+}\right]$ waves similar to those seen in SD.

\section{Materials and Methods}

Surgical procedure. All experiments procedures were performed according to the animal use and welfare guidelines of Rutgers University. Sprague Dawley rats [postnatal day 15 (P15) to P25)] were anesthetized with isoflurane $\left(5 \%\right.$ in an $\mathrm{O}_{2} / \mathrm{N}_{2} \mathrm{O}$ mixture for induction and $1-1.5 \%$ during the surgery; Attane; Minrad, Bethlehem, PA). Animals were tracheotomized and mechanically ventilated. The pulmonary volume was set, and blood gases were measured at the end of the experiment to avoid the effect of repetitive blood withdrawal $(80 \mu \mathrm{l})$ on arterial pressure 
( $\mathrm{PaCO}_{2}$ between 35 and $45 \mathrm{mmHg}, \mathrm{PaO}_{2}$ between 100 and $200 \mathrm{mmHg}$, $\mathrm{pH}$ between 7.35 and 7.50). The femoral artery was cannulated to permit vascular access and the monitoring of the mean arterial pressure (maintained between 75 and $100 \mathrm{mmHg}$ ). The rectal temperature was monitored and kept close to $37.5^{\circ} \mathrm{C}$ with a heating pad (Harvard Apparatus, Holliston, MA). A rectangular steel frame $(1.5 \times 6 \mathrm{~cm})$ with a hole $(4 \mathrm{~mm}$ diameter) centered over the somatosensory cortex was attached on the skull with dental cement. A craniotomy was performed, and the dura mater was removed. After the labeling procedure (below), the craniotomy was filled with agarose (type III-A, $0.75 \%$ in a HEPES-buffered solution; Sigma, St. Louis, MO) and covered with a coverslip immobilized with dental cement. After the surgery, isoflurane was discontinued, and the anesthesia was prolonged by intraperitoneal administration of ketamine/xylazine $(0.06$ and $0.01 \mathrm{mg} / \mathrm{g}$, respectively; Sigma) or chloral hydrate (10\%, $3 \mu \mathrm{l} / \mathrm{g}$; Sigma). Additional doses of choral hydrate $(2 \mu \mathrm{l} / \mathrm{g})$ were given as necessary. The first experiments were performed with ketamine/xylazine anesthesia, but, because of concerns over the possible effect of ketamine on SD, the anesthetic was switched to chloral hydrate, which was used for the vast majority of the experiments. Statistical testing for an effect of anesthesia on SD parameters, calcium transients, or vasoconstriction revealed no effect (data not shown); because of the difficulty of these experiments, the results below represent the pooled data from both regimens.

Labeling procedure. Two different $\mathrm{Ca}^{2+}$ indicator dyes were used (Invitrogen, Carlsbad, CA). For astrocyte-specific labeling, Fluo-4-AM [50 $\mu \mathrm{g}$ dissolved in $5 \mu \mathrm{l}$ of pluronic acid-DMSO (20\%) and $10 \mu \mathrm{l}$ of HEPES buffer, $\mathrm{pH}$ 7.42] was directly applied on the pial surface for $75 \mathrm{~min}$ and then washed with HEPES buffer. For bolus loading, Oregon Green BAPTA 1-AM (OGB-1) [50 $\mu \mathrm{g}$ dissolved in $5 \mu$ l of pluronic acid-DMSO $(20 \%)$ and $30 \mu \mathrm{l}$ of HEPES buffer] was pressure injected ( $200 \mathrm{nl}, 0.1-$ 0.2 bar in $10 \mathrm{~ms}$ pulses) with a micropipette $150-400 \mu \mathrm{m}$ below the surface. In all cases, astrocytes are labeled by bathing the cortical surface with a solution of $1 \mu \mathrm{M}$ sulforhodamine 101 (SR-101) (Texas Red; Sigma) for $10 \mathrm{~min}$. For vascular labeling, the femoral vein was cannulated for the injection of FITC dextran (molecular weight of $150,000,1 \%$ in saline, $100 \mu \mathrm{l})$.

$D C$ recording. An additional small hole was drilled over the frontal cortex with the dura mater intact, and a silver wire was inserted between the skull and the dura and immobilized with dental cement. The reference electrode was inserted superficially in the cerebellum. Alternatively, DC was recorded intracortically at the imaging site using a glass micropipette inserted through a partly open version of the cranial window. DC signals were amplified (DAM60; World Precision Instruments, Sarasota, $\mathrm{FL}$ ), digitized at $2 \mathrm{kHz}$ (Quad 16I, with DataTrax acquisition software; World Precision Instruments), and analyzed with custom software written in Matlab (MathWorks, Natick, MA).

Induction of spreading depression and anoxic depolarization. Spreading depression was induced by briefly pricking the cortex through an additional posterior hole with a needle (30 gauge) laced with $\mathrm{KCl}(0.1 \mathrm{M})$ and controlled by a micromanipulator. Cardiac arrest inducing anoxic depolarization was induced by air embolization: a bolus of $1 \mathrm{ml}$ of air was injected in the femoral vein.

Drug application. All drugs were diluted in HEPES buffer, $\mathrm{pH}$ 7.42. TTX $(100 \mu \mathrm{M})$, tetanus toxin (TeNT) $(1 \mu \mathrm{M})$, and $\mathrm{KCl}(300 \mathrm{~mm})$ combined with Texas Red were applied locally through a glass micropipette close to an intracortical arteriole (within $20-80 \mu \mathrm{m}$ ) at the lowest pressure that permitted the visualization of drug ejection on the red channel (1-10 psi in pulses of 10-30 ms). Injection of vehicle had no effect on vascular diameter or $\mathrm{Ca}^{2+}$ waves. Thapsigargin $(0.5 \mathrm{~mm})$ and methyl arachidonyl fluorophosphonate (MAFP) (1 mM) were applied directly to the surface of the exposed cortex for 20 min before the experiment.

Two-photon laser scanning microscopy. Imaging was performed using a custom-built two-photon laser scanning microscope consisting of an Olympus Optical (Melville, NY) trinocular, Cambridge Technology (Watertown, MA) scan mirrors, and a Thorlabs (Newton, NJ) scan lens, with a $20 \times$ water immersion objective lens (numerical aperture 0.95; Olympus Optical). Fluorescence emission was directed by a 700 longpass (LP) dichroic mirror, divided with a 565 LP dichroic mirror into green and red channels, and further restricted with BGG22 and
HQ607/45 filters placed before the green and red photomultiplier tubes (Hamamatsu, Bridgewater, NJ), respectively. The light source was a pulsed laser (Chameleon; Coherent, Santa Clara, CA) tuned to $810 \mathrm{~nm}$. Frames $(32 \times 32$ pixels) were collected continuously because it was impossible to predict precisely the arrival time of an SD wave. For linescanning experiments, a first SD was observed in frame-scan mode. Because consecutive SD waves are very likely to follow the same direction, a configuration of adjacent cells was chosen that was oriented orthogonally to the propagation direction, and a single line drawn through the center of all cell bodies was scanned. The inclusion of venules was avoided during imaging by following the penetrating trunk of a pial artery and by confirming the direction of the flow with a single line scan of small tributary or branch (supplemental Methods, available at www.jneurosci.org as supplemental material). The temperature inside the imaging chamber was kept close to $37^{\circ} \mathrm{C}$, with warm water flowing through tubing wrapped around the steel frame.

Data analysis. Neuron and astrocytes were distinguished using the red signal emitted by Texas Red. $\left[\mathrm{Ca}^{2+}\right]$ transients were measured by defined regions of interest inside of cell bodies. Signals were expressed as relative fluorescence changes $\left(\Delta F / F_{0}\right)$, where $F_{0}$ was the mean of the baseline period. The speed of $\left[\mathrm{Ca}^{2+}\right]$ waves was calculated by dividing the measurement of the distance between two cell bodies by the time lag between respective inflection points of $\left[\mathrm{Ca}^{2+}\right]$ transients. Arteriolar diameter change was analyzed with an automatic detection and measurement algorithm written in Matlab. Briefly, image sequences were binarized, and, if desired, the red channel was subtracted from the green channel to remove the green signal contributed by perivascular astrocytes. A line was drawn across the vessel (perpendicularly to the long axis for vessels imaged parallel to the $x-y$ plane or through the center for vessels imaged in cross section), and the number of pixels representing the vessel profile was computed along that line throughout the time series. The diameter in micrometers was then calculated based on the calibration of the microscope using beads of known sizes. For measurement of blood cell velocity, flux, and density, a capillary was imaged along its longitudinal axis in line-scan mode. Images were binarized and lines were fitted automatically to detect dark contours, which represent the profiles of unlabeled blood cells. Velocity was derived from the slope, and flux and density from the number, of detected contours as described previously (Dirnagl et al., 1992; Kleinfeld et al., 1998). Wave duration was defined as the time elapsed between the point at which the transient reached $50 \%$ of its maximal amplitude and the point at which it declined back to $50 \%$ of its maximal amplitude. Rise time was defined as the time required for the transient to increase from 10 to $90 \%$ of its maximal amplitude. Determination of the time of onset of astrocytic and neuronal waves from linescan data were made as follows: raw line-scan $\Delta F / F$ traces were filtered with a median filter. The mean and SD during a "baseline" period (minimum of $6000 \mathrm{~ms}$ ) were computed, and the onset of the transient was defined as the point that marked the beginning of a series of 300-500 consecutive points in which the $\Delta F / F$ value was $>2$ SDs above the baseline mean.

Statistics. The results are presented as mean \pm SEM. Statistical analyses were performed with an unpaired Student's $t$ test after the demonstration of homogeneity of variance with an $F$ test or a one-way ANOVA for more than two groups. The Bonferroni's test was used for post hoc testing. The $\alpha$ level was 0.05 (two-tailed).

\section{Results}

\section{$\left[\mathrm{Ca}^{2+}\right]$ waves during SD}

Astrocytes double-labeled in vivo with $\mathrm{Ca}^{2+}$-sensitive and $\mathrm{Ca}^{2+}$. insensitive dyes (Fluo-4 and SR-101, respectively) (Fig. 1a-d) displayed spontaneous $\left[\mathrm{Ca}^{2+}\right]$ transients as described previously (Hirase et al., 2004). These low-frequency transients $(5.9 \pm 0.5 \times$ $10^{-3} \mathrm{~Hz} ; 144$ astrocytes from 8 rats), seen in $75 \%$ of astrocytes, were usually restricted to individual astrocytes, but some appeared to propagate from astrocyte to astrocyte. When SD was induced with a pinprick laced with $\mathrm{KCl}$, two types of wavelike transients were elicited (Fig. $1 e-g$ ). One was of long duration, relatively slow rise time, and slower propagation speed $(6.1 \pm 0.7$ 
$\mu \mathrm{m} / \mathrm{s} ; n=13$ SDs from 12 animals). The other was of short duration, very short rise time, and faster propagation speed ( $35 \pm 2$ $\mu \mathrm{m} / \mathrm{s} ; n=37$ SDs from 25 animals). The slow waves were observed in only $35 \%$ of SDs, whereas fast waves always accompanied SDs. Slow waves were multifocal with a variable direction of propagation and did not involve all astrocytes $(82 \pm 8 \%$ of astrocytes; $n=13$ SDs from 12 animals). Fast waves had a constant direction of propagation within an animal and involved $100 \%$ of astrocytes. In most cases, slow waves preceded the pass of the fast wave $(66.0 \% ; n=13)$.

To determine whether neurons also participate in these propagating signals, OGB-1 was introduced into the cortex using the bolus-loading technique (Stosiek et al., 2003) (Fig. 2a). Neurons were distinguished from astrocytes by the selective labeling of astrocytes with SR-101 (Nimmerjahn et al., 2004) (Fig. 2b,c). Astrocytes manifested the same two waves as seen with Fluo-4. Neurons showed one wave that corresponded temporally and kinetically only with the faster astrocytic wave (Fig. $2 d, e$ ). Because SD in vitro has been associated with $\left[\mathrm{Ca}^{2+}\right]$ transients in neurons (Peters et al., 2003), we hypothesized that this fast wave represents the SD wave itself. To address the question of whether astrocytes lead or follow in the propagation of SD, we analyzed the time of onset of the fast $\left[\mathrm{Ca}^{2+}\right]$ transients in neighboring astrocytes and neurons to determine whether the wave occurs first in neurons or in astrocytes. At our imaging frequency $(1.4 \mathrm{~Hz})$, many astrocytic and neuronal waves could not be temporally resolved. However, in the cases in which they could (17 of 27 cases in 5 animals), analysis of the lag showed that they occurred first in neurons $88 \%$ of the time. Because our sampling frequency was inadequate to reliably resolve differences under $\sim 1 \mathrm{~s}$, we used line scans, increasing our sampling frequency to $500 \mathrm{~Hz}$. In 20 astrocyte-neuron pairs from eight animals in which a fortuitous arrangement of neurons and astrocytes allowed us to perform this measurement (Fig. $2 f-h)$, a neuronal $\left[\mathrm{Ca}^{2+}\right]$ transient preceded that in a neighboring astrocyte in $75.0 \%$ of the cases (Fig. $2 i$ ). The neuronal and astrocytic transients were tightly coupled, with a median lag (in either direction) of $\sim 300 \mathrm{~ms}$. The $10-90 \%$ rise time of neuronal and astrocytic $\left[\mathrm{Ca}^{2+}\right]$ transients were not significantly different $(4.1 \pm 1.0$ and $3.4 \pm 0.7 \mathrm{~s}$, respectively) and correlated with each other within each experiment $(r=0.91)$, sug-
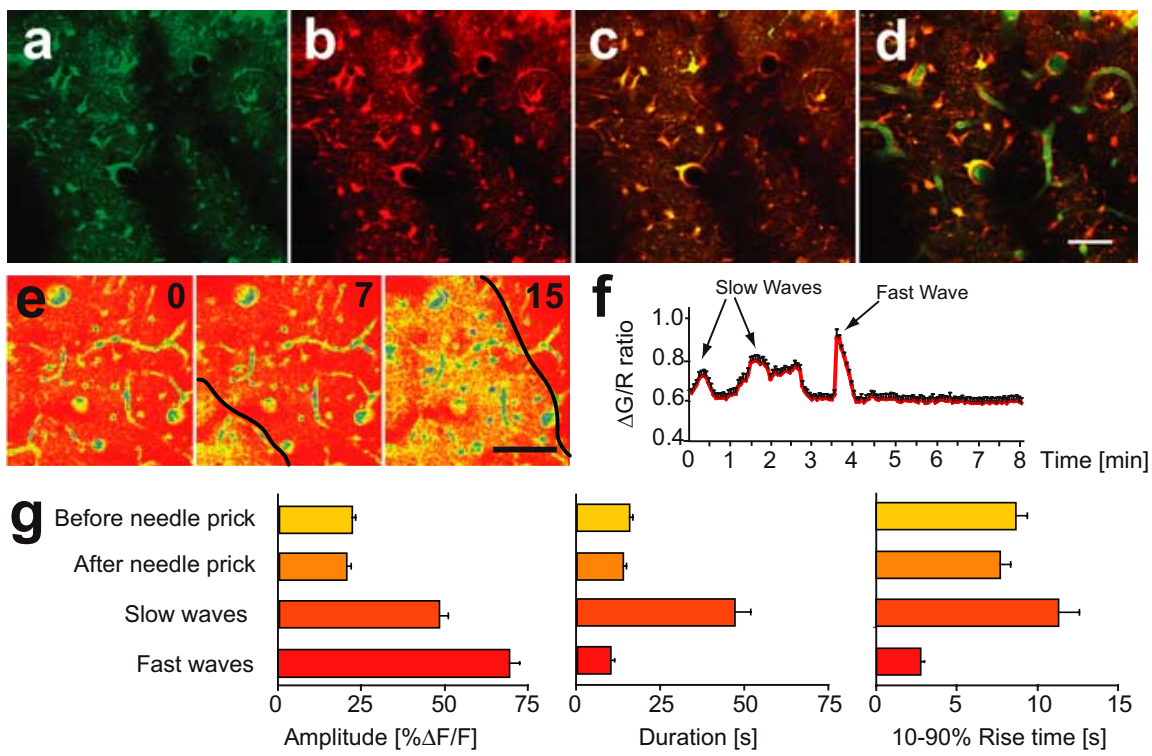

Figure 1. SD-associated $\left[\mathrm{Ca}^{2+}\right]$ waves in astrocytes in vivo. Astrocytes were labeled with a combination of Fluo-4 (a) and SR-101 ( $\boldsymbol{b}$; merged in $\boldsymbol{c}$ ). Infusion of FITC dextran labeled the vasculature (d). Scale bar, $50 \mu \mathrm{m}$. An SD wave is shown in $\boldsymbol{e}$; the wave front is indicated by the black line. Time stamps are in seconds. Scale bar, $100 \mu \mathrm{m}$. The change in astrocytic $\left[\mathrm{Ca}^{2+}\right]$ is plotted in $f$ (mean \pm SEM over 13 astrocytes). $\boldsymbol{g}$, Spontaneous waves before and after the needle prick differed from both slow and fast SD-associated waves amplitude, duration, and rise time.
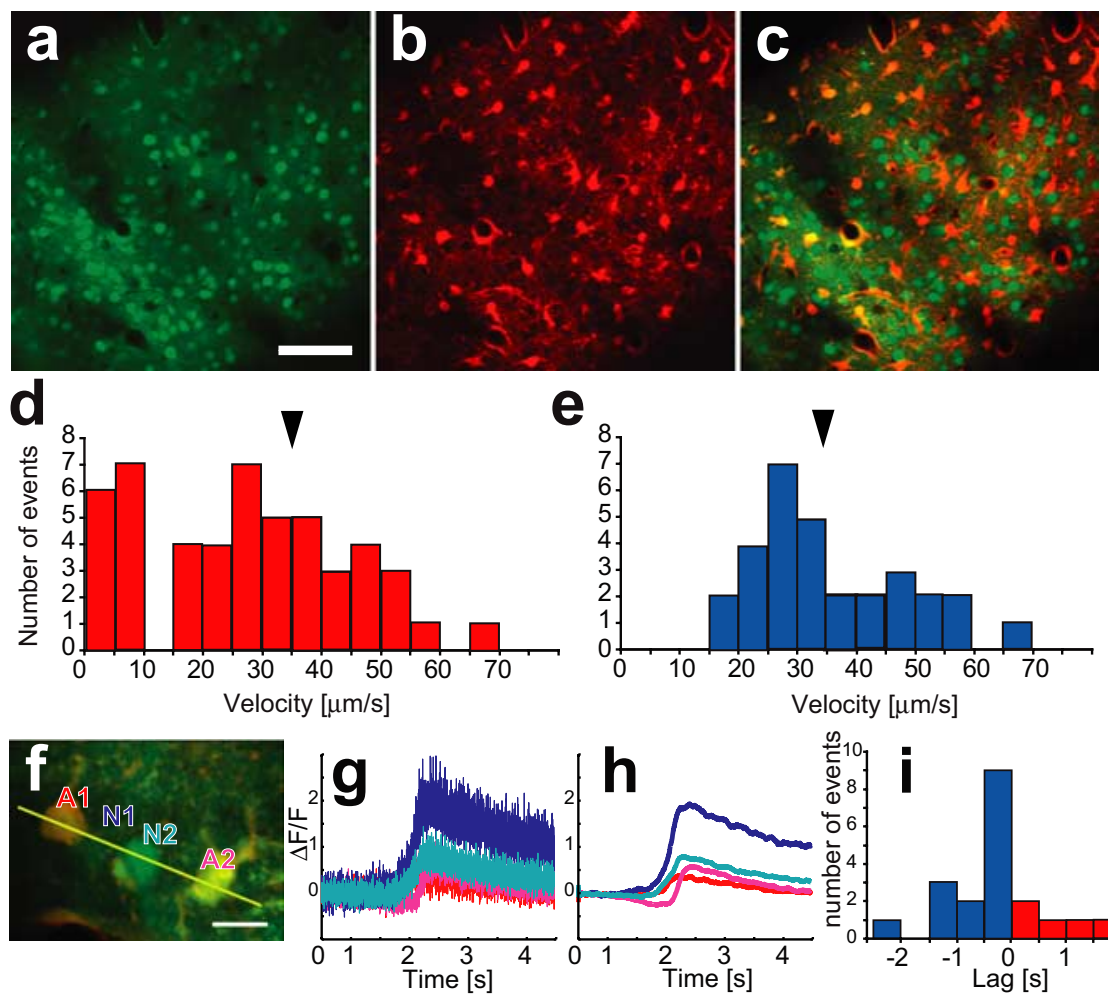

$\checkmark$
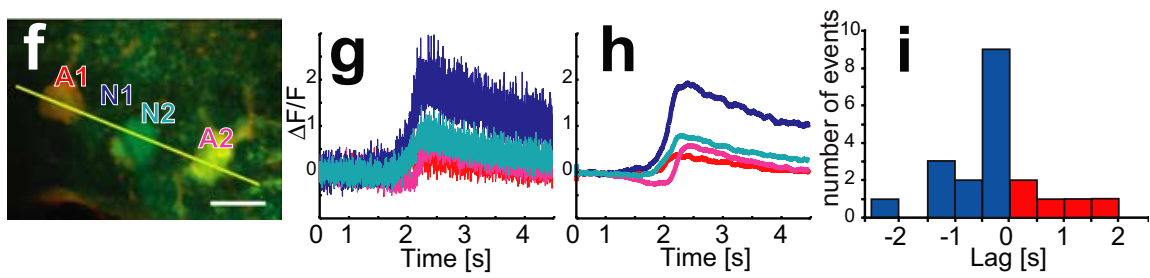

Figure 2. SD-associated $\left[\mathrm{Ca}^{2+}\right]$ waves in neurons in vivo. Astrocytes and neurons were labeled with 0GB-1 (a), and astrocytes were also labeled with SR-101 $(\boldsymbol{b})$. Green and red channels are merged in $\boldsymbol{c}$; note that neurons are labeled only with $0 \mathrm{~GB}-1$, whereas astrocytes are double labeled as in Figure 1, permitting their identification. Scale bar, $100 \mu \mathrm{m}$. Astrocytic [Ca $\left.{ }^{2+}\right]$ waves plotted by velocity of propagation showed a broad distribution with slow $(<10 \mu \mathrm{m} / \mathrm{s})$ and faster $(>15 \mu \mathrm{m} / \mathrm{s})$ waves $(\boldsymbol{d})$, whereas the neuronal distribution included no waves slower than $15 \mu \mathrm{m} / \mathrm{s}(\boldsymbol{e})$. Arrowheads indicate the mean velocity of waves associated with vasoconstrictions. Line-scan experiments of nearby astrocytes ( $\boldsymbol{f} ; A 1, A 2)$ and neurons (N1, N2) showed that the $\left[\mathrm{Ca}^{2+}\right]$ wave usually occurred first in neurons. Scale bar, $20 \mu \mathrm{m} . \boldsymbol{g}$, Raw line-scan data for the four cells indicated in $\boldsymbol{f}$. $\boldsymbol{h}$, Same data low-pass filtered to show onsets of the relatively slow waves. $i$, Summary data for 20 neuron-astrocyte pairs. The lag is expressed as the time when the neuronal transient was detected minus the time when the astrocytic transient was detected (in seconds). Note that the majority of lags were $<500$ ms in either direction. 

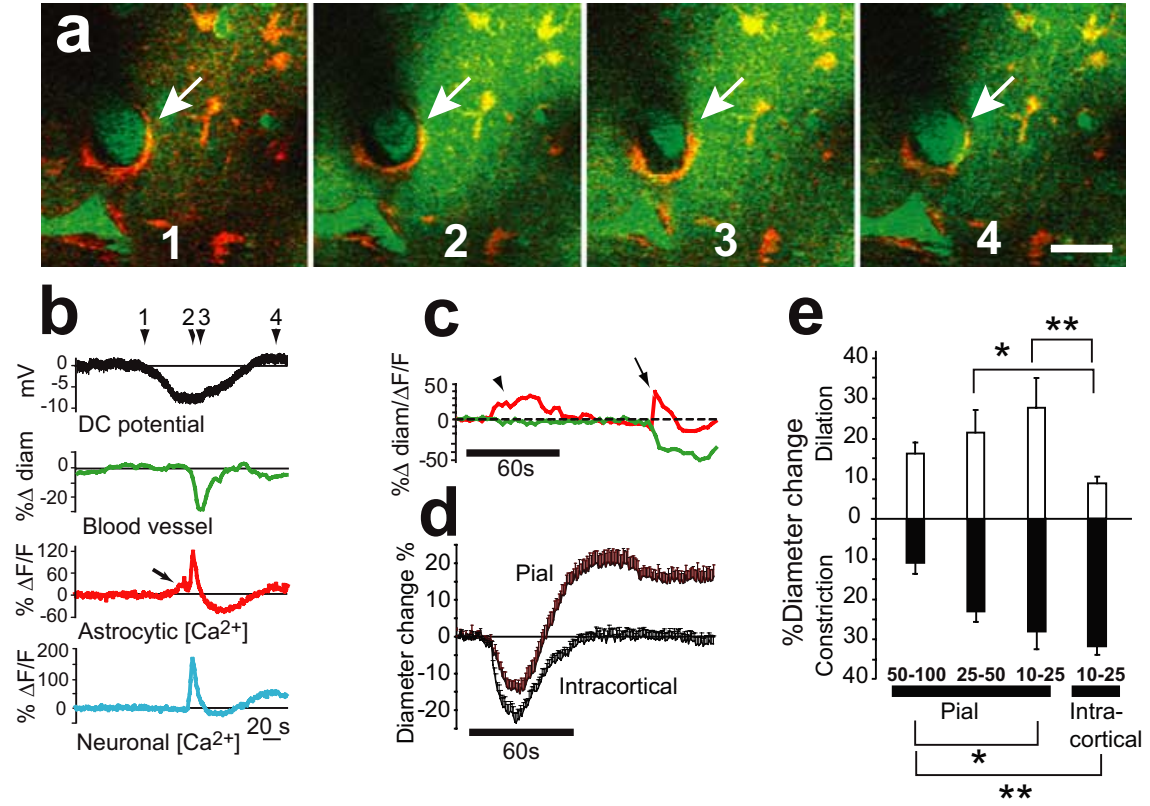

Figure 3. Vascular dynamics in SD. Penetrating intracortical arteries constricted dramatically during SD ( $\boldsymbol{a}$; numbers indicate time points marked in $\boldsymbol{b}$ ). $\boldsymbol{a}, \boldsymbol{b}$, Cerebral vessels were imaged by the intravascular introduction of FITC dextran, enabling measurement of changes in vascular diameter as well as in capillary perfusion. Scale bar, $20 \mu \mathrm{m}$. $\boldsymbol{b}$, Correlation of DC potential with vasoconstriction, and astrocytic and neuronal $\left[\mathrm{Ca}^{2+}\right]$ waves. Note the slow astrocytic $\left[\mathrm{Ca}^{2+}\right]$ wave colliding with the fast wave in c that was not associated with vascular change. In this example, a DC potential was recorded with a subdural electrode. $c$, Slow astrocytic $\left[\mathrm{Ca}^{2+}\right]$ waves (arrowhead) were not associated with vasoconstriction, whereas fast ones (arrow) were. This example was taken from a different animal from that shown in $\boldsymbol{a}$ and $\boldsymbol{b}$. $\boldsymbol{d}$, Pial arterioles showed a biphasic response, with initial constriction followed by a long-lasting dilation. Intracortical arterioles ( $n=29$ from 15 animals) showed only a constriction that returned to baseline. As with intracortical arterioles, the onset of pial vasoconstriction was spatiotemporally correlated with the calcium wave. $\boldsymbol{e}$, Peak diameter change varied with vessel location and caliber. Pial arteries showed constriction and dilation that were inversely related to caliber, whereas small intracortical arteries notably lacked a considerable dilatory response, in contrast to comparably sized pial arterioles ( $n=6$ from 4 animals). Constriction of large pial arteries $(50-100 \mu \mathrm{m} ; n=5$ from 4 animals) was significantly smaller than that in other arteries, and intracortical small arteriolar dilation differed significantly from dilation of small $(10-25 \mu \mathrm{m})$ and medium $(25-50 \mu \mathrm{m})$ pial arteries $\left(n=8\right.$ from 3 animals). ${ }^{*} p<0.05{ }^{* *} p<0.001$.

gesting that the lag could not be attributed to the kinetics of the $\left[\mathrm{Ca}^{2+}\right]$ rise in the two cell types.

\section{Correlation between $\left[\mathrm{Ca}^{2+}\right]$ waves and vasoconstriction}

Spontaneous transients or waves were not associated with vasodilation or vasoconstriction (mean change, $-0.28 \pm 0.72 \% ; n=$ $13 ; p=0.26$ ), even when the transient invaded perivascular end feet (supplemental Video 1, available at www.jneurosci. org as supplemental material). Similarly, the slow, purely astrocytic transients (velocity $<10 \mu \mathrm{m} / \mathrm{s}$ ) were never associated with a significant change in arteriolar diameter $(-1.8 \pm 0.9 \% ; n=6$ waves from 6 animals; $p=0.21$ ) (supplemental Video 2, available at www.jneurosci.org as supplemental material). Intracortical arteries were not in their maximal state of dilation because they responded to a hypercapnic test $\left(5 \% \mathrm{CO}_{2}\right.$ for $\left.20 \mathrm{~s}\right)$ with an increase of $8.1 \pm 0.8 \%$ of their diameter $(n=10$ arteries from 4 animals; $p \ll 0.001$ ). In contrast, the fast calcium waves (velocity $>15 \mu \mathrm{m} / \mathrm{s}$ ) were always spatiotemporally correlated with vasoconstriction (see below) (Fig. 3a-c) (supplemental Videos 2, 3, available at www.jneurosci.org as supplemental material).

\section{Cerebrovascular effects of SD}

Pial arteries showed a biphasic pattern of vasomotion consisting of a sharp constriction at the onset of the $\left[\mathrm{Ca}^{2+}\right]$ wave, followed by a longer-lasting dilation (Fig. $3 d$ ). They responded in a caliberdependent manner (Fig. 3e). Major trunks of the middle cerebral

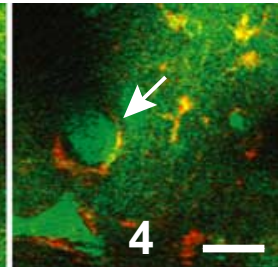

artery $(50-100 \mu \mathrm{m})$ displayed a slight constriction, followed by a maximal dilation of $16 \pm 3 \%$. For intermediate pial arteries $(25-50 \mu \mathrm{m})$, constriction and dilation were more pronounced. The smallest pial arterioles $(10-25 \mu \mathrm{m})$ showed the greatest diameter change with $28 \pm 4 \%$ of constriction, followed by a dilation of $28 \pm 7 \%$. Penetrating arteries as well as intracortical arterioles underwent a dramatic constriction $(-31 \pm 2 \%)$ beginning at the onset of the spreading $\left[\mathrm{Ca}^{2+}\right]$ wave, recovered to the baseline within $3 \mathrm{~min}$, and had a small, variable dilation (mean maximal dilation, $9 \pm 1 \%$ ) (Fig. 3e). Intracortical venules did not constrict with the onset of the wave but showed a small but significant constriction $(2.8 \pm 0.6 \% ; p=$ $0.003) 22 \pm 3 \mathrm{~s}$ after the peak of the wave.

Capillary perfusion, which was stable over a control period of at least $5 \mathrm{~min}$ (Fig. $4 a-c$ ) (mean velocity, $1.2 \pm 0.2 \mathrm{~mm} / \mathrm{s}$; mean flux, $41 \pm 5$ cells/s), was dramatically affected by SD. During the spread of the $\left[\mathrm{Ca}^{2+}\right]$ wave, blood cell velocity dropped by $87 \pm 4 \%(n=20)$ (supplemental figure, available at www. jneurosci.org as supplemental material). In $19 \pm 4 \%$ of capillaries observed (139 observations from 15 animals), there was a cessation of flux that lasted for $69.5 \pm 14.5 \mathrm{~s}$ (Fig. 4e). The duration of this ischemia was significantly correlated $(r=0.836 ; p<0.001)$ with the initial level of perfusion (Fig. $4 f, g$ ). The capillary lumen was always visualized, and no "string vessels" were observed, indicating that there was no local capillary collapse.

In these experiments, we seldom imaged long enough to detect the long-lasting oligemia that is well described in SD (Fabricius et al., 1995). However, in three animals in which experiments continued $7 \mathrm{~min}$ after induction of SD, we observed a nonsignificant tendency of intracortical arteries to decrease in diameter $(-2.7 \pm 1.9 \% ; p=0.24)$. In one case of long-term imaging, we observed a change of $-9 \%$ in the diameter at 18 min after the onset of SD. However, because these results were not systematically obtained, were highly variable, and were not temporally correlated with any $\left[\mathrm{Ca}^{2+}\right]$ waves, we did not emphasize them in this study.

\section{Agents of SD-induced vasoconstriction}

To investigate whether the astrocytic $\left[\mathrm{Ca}^{2+}\right]$ wave plays a role in the vasoconstriction, we interfered with it pharmacologically. Because most astrocytic $\left[\mathrm{Ca}^{2+}\right]$ transients and waves are mediated by release of $\mathrm{Ca}^{2+}$ from internal stores (Giaume and Venance, 1998), we applied thapsigargin, a sarcoplasmic/endoplasmic reticulum $\mathrm{Ca}^{2+}$-ATPase inhibitor that prevents refilling of internal $\mathrm{Ca}^{2+}$ stores. Whereas the neuronal signal was unaffected in amplitude, slope, and duration, the astrocytic signal was markedly reduced in all three parameters (Fig. $5 a-c)(\Delta F / F$ amplitude, $-47.2 \%, p \ll 0.001$; duration, $-64.6 \%, p \ll 0.001 ; 10-90 \%$ rise time change, $-34.5 \%, p=0.004)$. Vasoconstriction was inhibited in the presence of thapsigargin $(-51 \% ; p=0.003)$ (Fig. 

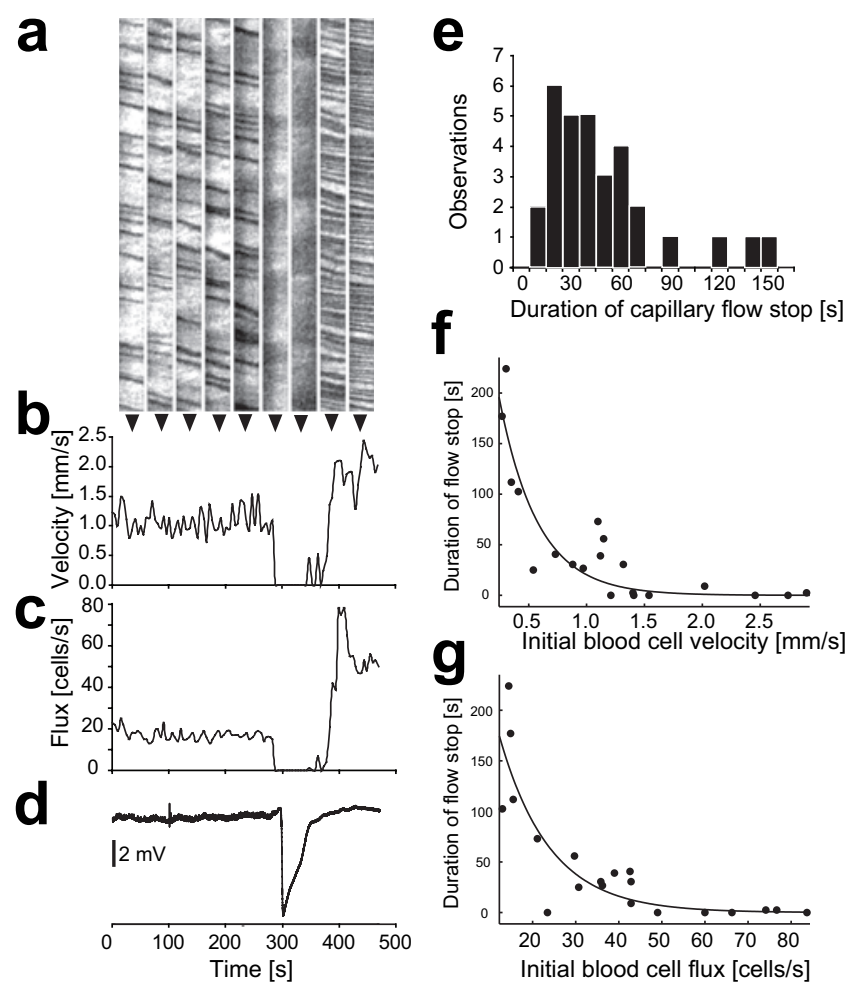

Figure 4. Capillary blood flow dynamics in SD. Capillary blood flow slowed dramatically (a) and occasionally ceased completely $(\boldsymbol{b}, \boldsymbol{c})$ in SD. $\boldsymbol{a}$, Line scans taken at the time points indicated by the arrowheads. Note the rebound increase in blood flow after SD (2 rightmost scans). Velocity $(\boldsymbol{b})$ and flux (c) were fairly stable in baseline and then, in this example, dropped to 0 during SD. DC recording (intracortical electrode $300 \mu \mathrm{m}$ from imaging site) is shown in $\boldsymbol{d} . \boldsymbol{e}_{\text {, }}$ Distribution of duration of capillary flow cessation in cases in which flow stopped completely. Duration of blood flow stop was negatively correlated with the initial blood cell velocity $(\boldsymbol{f})$ and flux $(\boldsymbol{g})$.

$5 d, e)$, suggesting that astrocytic $\left[\mathrm{Ca}^{2+}\right]$ waves are instrumental in mediating SD-induced vasoconstriction.

Although in view of the above and a recent study (Mulligan and MacVicar, 2004), it is tempting to attribute SD-induced vasoconstriction to the large astrocytic $\left[\mathrm{Ca}^{2+}\right]$ waves, $\mathrm{SD}$-induced vasoconstriction could be attributable to a number of mechanisms. First, during SD, all neurons depolarize, including those that influence vascular tone (Cauli et al., 2004). Local application of TTX did not, however, affect the presence, kinetics, or propagation speed of the SD wave in astrocytes. It prolonged the mean duration of the neuronal transient but had no effect on transient amplitude, rise time, or propagation speed (Fig. $5 a-c$ ). Importantly, it did not diminish vasoconstriction $(-23 \pm 0.4$ vs $-24 \pm$ $3 \% ; p=0.30)$. Because neuronal depolarization in SD does not necessarily involve voltage-gated $\mathrm{Na}^{+}$channels (Somjen, 2001), the effect of TeNT was tested as well. Short ( $\sim 30 \mathrm{~min})$ exposures to TeNT selectively impair neuronal exocytosis; very long exposures $(\sim 8 \mathrm{~h})$ are necessary to block astrocytic exocytosis (Bezzi et al., 1998; Pasti et al., 2001). After 10-20 min exposure to TeNT, astrocytic $\left[\mathrm{Ca}^{2+}\right]$ transients were unaffected, except for an increase in their rise time ( $5.2 \pm 0.6$ vs $2.7 \pm 0.2 s ; p=0.0001$ ) (Fig. $5 c$ ). Neuronal $\left[\mathrm{Ca}^{2+}\right]$ transients also had a slower rise time (5.7 \pm 0.6 vs $2.8 \pm 0.2 \mathrm{~s} ; p \ll 0.0001$ ) (Fig. $5 c$ ) and were of longer duration (14.0 \pm 1.5 vs $5.5 \pm 0.3 \mathrm{~s} ; p \ll 0.0001$ ) (Fig. $5 b$ ). These changes suggest that neurotransmitter release plays a role, at least, in determining the kinetics of SD-associated $\left[\mathrm{Ca}^{2+}\right]$ transients. However, SD-associated vasoconstriction was unaffected
$(-25 \pm 3$ vs $-24 \pm 3 \% ; p=0.80 ; n=6 \mathrm{SD}$ in 3 animals), suggesting that this aspect of $\mathrm{SD}$ is not mediated by neuronal vesicular neurotransmitter release.

Alternatively, the propagating wave of $\mathrm{K}^{+}$itself could be responsible, because $\mathrm{K}^{+}$is a known constrictor of extracortical vessels (Faraci and Sobey, 1998), although its effects on intracortical arterioles in vivo are unknown. To test this hypothesis, we applied $\mathrm{KCl}$ locally to cortical arterioles under optical guidance (Fig. 5f). $\mathrm{KCl}$ caused a vasoconstriction (diameter, $-16 \pm 4 \%$; $n=9$ from 3 animals), which was insensitive to thapsigargin $(-18 \pm 2$ vs $-15 \pm 3 \% ; p=0.62)$ (Fig. $5 g)$. This vasoconstriction was not accompanied by a DC shift as measured via an electrode positioned within the imaging window, indicating that the injection of this small volume of $\mathrm{KCl}(\sim 75 \mathrm{nl})$ was insufficient to cause a regenerative $\mathrm{SD}$ ( $n=9$ injections in 3 animals). Because thapsigargin reduced SD-induced vasoconstriction (Fig. $5 d-g$ ), this suggests a mechanistic difference between $\mathrm{SD}$ - and $\mathrm{K}^{+}$-induced vasoconstriction. Moreover, release of $\mathrm{Ca}^{2+}$ from internal stores is not required for vasoconstriction in these arteries, as suggested in a previous study (Flemming et al., 2002), and the thapsigargin block of vasoconstriction in SD was not attributable to a direct effect of thapsigargin on arterioles.

Astrocytic $\left[\mathrm{Ca}^{2+}\right]$ has been shown in vitro to cause vasoconstriction via the phospholipase A-mediated release of arachidonic acid (Mulligan and MacVicar, 2004). To strengthen further the link between the astrocytic $\left[\mathrm{Ca}^{2+}\right]$ rise and vasoconstriction, MAFP, an inhibitor of the calcium-dependant phospholipase A2 (Street et al., 1993) was administered before induction of SD. Under these conditions, although the $\left[\mathrm{Ca}^{2+}\right]$ wave was unaffected (amplitude, $71.8 \pm 2.8$ vs $69.3 \pm 2.9 \%, p=0.33$; duration, $7.6 \pm 0.5$ vs $10.0 \pm 1 \mathrm{~s}, p=0.12$; rise time, $2.0 \pm 0.2$ vs $2.7 \pm 0.2 \mathrm{~s}$, $p=0.06)$, vasoconstriction was markedly reduced, from $-24 \pm$ 3 to $-9 \pm 1 \%(p \ll 0.0001 ; n=10$ arterioles in 3 animals) (Fig. $5 h, i)$.

\section{Anoxic depolarizations}

$\mathrm{AD}$, which resembles $\mathrm{SD}$ electrophysiologically, precedes cell damage in the compromised brain (Jarvis et al., 2001). Although $\mathrm{SD}$ is a useful model for the changes that occur with ischemia, it is an imperfect one, partly because it does not cause cell death (Nedergaard and Hansen, 1988). We therefore induced cardiac arrest by air embolization to determine whether the cellular events we observe in SD occur also in AD. A few minutes $(380 \pm 49 \mathrm{~s} ; n=$ 3 ) after the cessation of blood flow as viewed by imaging blood vessels, we detected a DC shift accompanied by both astrocytic and neuronal $\left[\mathrm{Ca}^{2+}\right]$ transients (Fig. $6 a, b$ ) reminiscent of those seen in SD (Fig. 6c-d). The velocity of this wave $(27 \pm 3 \mu \mathrm{m} / \mathrm{s}$; $n=3$ ) was lower than the velocity of the SD wave, albeit not significantly $(35 \pm 2 ; n=25 ; p=0.13$ ) (Fig. $6 e-g)$. However, the amplitude of the transient was significantly greater in $\mathrm{AD}$ than in $\mathrm{SD}$ in both astrocytes $(+211 \% ; p \ll 0.0001)$ and neurons $(+215 \% ; p \ll 0.0001)$, whereas the slope of the transient $(10-$ $90 \%$ rise time) was significantly larger in $\mathrm{AD}$ than in $\mathrm{SD}$ for both cell types $(+362 \%, p=0.0002$ for astrocytes and $+483 \%, p \ll$ 0.0001 for neurons). Furthermore, whereas intracellular $\left[\mathrm{Ca}^{2+}\right]$ in $\mathrm{SD}$ returned to baseline within $1 \mathrm{~min}$, in $\mathrm{AD}$ it remained elevated for $\geq 3 \min$ ( $106.1 \pm 7.7 \%$ for neurons and $75.0 \pm 9.3 \%$ for astrocytes, above baseline). Although blood flow arrest was observed under these conditions, because $\mathrm{AD}$ was induced with global ischemia via cardioplegia, one would fully expect there to be a range of vascular responses that could just as easily be attributable to responses to passive collapse when perfusion pressure drops below the threshold for autoregulation. This would com- 

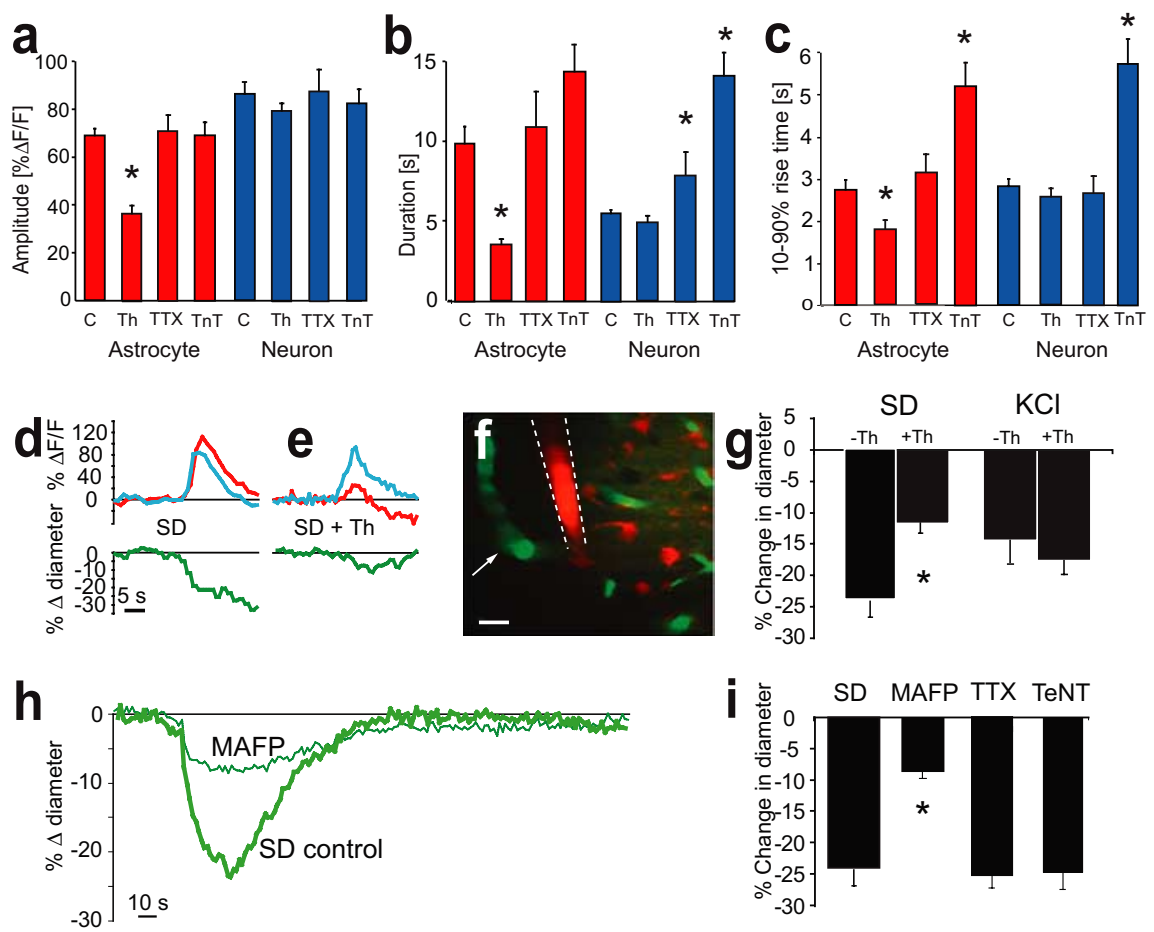

Figure 5. Thapsigargin selectively reduced astrocytic $\left[\mathrm{Ca}^{2+}\right]$ waves and blunted SD-associated vasoconstriction. Fast astrocytic $\left[\mathrm{Ca}^{2+}\right]$ waves were reduced in amplitude $\left(\boldsymbol{a}^{*}{ }^{*} p \ll 0.001\right)$, duration $\left(\boldsymbol{b} ;{ }^{*} p=0.007\right)$, and rise time $\left(\boldsymbol{c} ;{ }^{*} p \ll 0.001\right)$ in the presence of thapsigargin ( $n=50$ astrocytes from 3 animals). Neuronal [ $\mathrm{Ca}^{2+}$ ] waves were unaffected ( $n=36$ neurons from 3 animals). In contrast, TTX had no effect on astrocytic waves but increased the duration of neuronal $\left[\mathrm{Ca}^{2+}\right]$ waves $(n=19$ astrocytes and $n=15$ neurons from 3 animals). Tetanus toxin had no effect on astrocytic or neuronal [ $\left.\mathrm{Ca}^{2+}\right]$ wave amplitude but prolonged neuronal $\left[\mathrm{Ca}^{2+}\right]$ waves and slowed the rise time of astrocytic and neuronal $\left[\mathrm{Ca}^{2+}\right]$ waves $(n=49$ astrocytes and $n=$ 56 neurons from 3 animals). C, Control; Th, thapsigargin; TnT, tetanus toxin. $\boldsymbol{d}, \boldsymbol{e}$, Astrocytic (red) and neuronal (blue) $\left[\mathrm{Ca}^{2+}\right]$ waves and vasoconstriction (green) in the absence $(\boldsymbol{d})$ and presence $(\boldsymbol{e})$ of thapsigargin. $\boldsymbol{f}, \boldsymbol{g}$, SD-associated vasoconstriction was not mediated by neuronal depolarization or direct vascular depolarization by $\mathrm{K}^{+} . \boldsymbol{f}, \mathrm{KCl}$ application via a micropipette (filled with SR-101; dotted lines) constricted arterioles (arrow) in both the presence and absence of thapsigargin, in contrast to SD, whose vasoconstriction was reduced by thapsigargin. Scale bar, $20 \mu \mathrm{m}$. $\boldsymbol{g}$, Summary data of SD and KCl vasoconstriction in the absence ( $n=26$ arteries from 12 animals and $n=9$ arteries from 3 animals, respectively) and presence ( $n=12$ arteries from 4 animals and $n=8$ arteries from 3 animals, respectively) of thapsigargin $\left({ }^{*} p=0.009\right)$. $\boldsymbol{h}, \mathrm{SD}$-associated vasoconstriction (thick line) is inhibited by topically applied MAFP (thin line). $\boldsymbol{i}$, Summary data of SD-associated vasoconstriction in the absence and presence of MAFP. ( ${ }^{*} p \ll 0.0001 ; n=29$ arterioles in 15 animals and $n=10$ arterioles in 3 animals, respectively)

plicate interpretation, and not necessarily add to the findings of the present study, and therefore the details of this vascular change were not studied further.

\section{Discussion}

We describe a vascular response to SD in vivo that can halt blood flow for more than $1 \mathrm{~min}$. This vasoconstriction could have several possible explanations. First, during SD, there is swelling of the brain in general, which could produce a passive apparent vasoconstriction (Tomita et al., 2005). However, veins of comparable caliber were affected much less, despite their low perfusion pressure and thinner walls, and considerably later than arterioles. Thus, although tissue swelling could contribute to the later phase of the capillary blood flow arrest, it does not explain it. Second, there might be a direct effect of $\mathrm{K}^{+}$on vascular diameter. Application of $\mathrm{KCl}$ to intracerebral arterioles caused vasoconstriction that, however, differed from that seen in SD in its sensitivity to thapsigargin. Third, because SD depolarizes all neurons, vasoconstriction could be attributable to spiking in neurons innervating the vasculature (Cauli et al., 2004). The absence of an effect of either TTX or TeNT makes this unlikely. We are left, then, with a primarily astrocyte-mediated effect. Astrocytic $\left[\mathrm{Ca}^{2+}\right]$ transients have been shown to cause vasoconstriction (Mulligan and MacVicar, 2004) or vasodilation in vitro (Zonta et al., 2003; Metea and Newman, 2006) and in vivo (Takano et al., 2005). The blockade of vasoconstriction when astrocytic $\left[\mathrm{Ca}^{2+}\right]$ waves were diminished with thapsigargin, and the inhibitory effect of MAFP on vasoconstriction, support some of these findings (Mulligan and MacVicar, 2004). However, even large and/or prolonged astrocytic $\left[\mathrm{Ca}^{2+}\right]$ transients in the absence of SD had no effect on arteriolar diameter, and neither did the slow $\left[\mathrm{Ca}^{2+}\right]$ waves that occasionally occurred after SD induction. The ability of an astrocytic $\left[\mathrm{Ca}^{2+}\right]$ transient to cause vascular change may be a function of the properties of the transient, such as its amplitude, rate of onset or decay, or its $\mathrm{Ca}^{2+}$ source.

Perivascular astrocytes are exposed to the same $\mathrm{K}^{+}$as their neighboring vessels. It is therefore significant that it was possible to tease apart the two phenomena. This suggests that the response of astrocytes to $\mathrm{SD}$ is not the immediate result of their detection of high $\left[\mathrm{K}^{+}\right]_{\mathrm{e}}$, but that there are steps intervening between the elevated $\left[\mathrm{K}^{+}\right]_{\mathrm{e}}$ and astrocyte-mediated vasoconstriction.

We describe two $\left[\mathrm{Ca}^{2+}\right]$ waves distinguishable by their propagation speed, incidence, and their astrocytic and neuronal involvement. Unlike the fast wave, the slow wave propagates much slower than $\mathrm{SD}$, occurs in only $35 \%$ of SDs, and does not involve all astrocytes or any neurons. It thus differs substantially from what one would expect from a phenomenon that is an integral part of SD. We regard these as astrocytic $\left[\mathrm{Ca}^{2+}\right]$ waves, as seen in vitro (Cornell-Bell et al., 1990; Charles et al., 1991; Wang et al., 1997), expressed as a collateral phenomenon of SD but whose origin and significance are unclear.

The 15-25\% pial arteriolar dilation described here translates into a $75-140 \%$ increase in flow, according to Poiseuille's law, assuming constant blood pressure. This change is well within the range of estimates of SD-associated blood flow increases using LDF [43-154\% increase (Back et al., 1994; Lauritzen and Fabricius, 1995; Fabricius et al., 1997; Gold et al., 1998; Nielsen et al., 2000)] or laser speckle [60-150\% increase (Dunn et al., 2001)], supporting the validity of our technique and suggesting that blood pressure is not altered immediately upstream of our imaging site.

Most previous studies support a biphasic hemodynamic change in SD, with a large hyperperfusion shortly after the DC shift followed by a long-lasting hypoperfusion (Goadsby, 1992; Lacombe et al., 1992; Seitz et al., 2004). Some studies also reported that, before the hyperemic phase, there is a transient hypoperfusion (Dreier et al., 2001; Ayata et al., 2004; Tomita et al., 2005). We focus only on the acute changes that accompany the DC shift and not on the oligemia that occurs later and may last hours (Goadsby, 1992; Lacombe et al., 1992). We demonstrate 
that, after the constriction of cerebral arterioles, the hyperemic phase is mainly supported by the dilation of the pial arterioles. This difference might be attributable to the differential investment of pial and intracortical vessels with astrocytes (Peters et al., 1991) or a differential innervation by autonomic and sensory ganglia (Iadecola, 2004).

One explanation for the discrepancy between this and previous studies likely lies in the way signals are obtained using LDF. In LDF, a large area $\left(\sim 1 \mathrm{~mm}^{2}\right.$; total volume, $\sim 1 \mathrm{~mm}^{3}$ ) is imaged at once, and the signal across that whole region is averaged. Changes in very small compartments, such as small intracortical arterioles, are minimized under these conditions, particularly if they occur only in a small proportion of the field. Conversely, changes in the largest compartments, such as large pial vessels, will be relatively predominant, a bias that is exacerbated by the fact that the LDF signal is strongly weighted toward the cortical surface. With the present imaging method, the area scanned is much smaller $(\sim 0.04$ $\mathrm{mm}^{2}$; total volume, $\sim 0.00008 \mathrm{~mm}^{3}$ ), and measurements are not influenced by vessel size because arterioles are imaged individually and signals are not averaged over entire fields. This method is therefore more sensitive to changes in the smallest compartments and not unduly influenced by changes in the largest ones.

Another difference between this study and previous ones is the age of the animals. The present experiments were performed in juvenile rats (P15-P25), because intracellular loading for the calcium and astrocytic imaging with SR-101 decreases in efficiency after this age (Hirase et al., 2004; Nimmerjahn et al., 2004). Spreading depression arises in animals during the second postnatal week and is well developed by P15 (Bures, 1957; de Luca and Bures, 1977), the earliest age used here. However, its kinetics continue to change for several weeks (de Luca and Bures, 1977), and this could contribute to differences between these findings and those obtained in older rats.

There is a species discrepancy in the literature, in which vasoconstrictive coupling was observed in SD in mice but not in adult rats using LDF (Ayata et al., 2004; Shin et al., 2006). This study might help resolve this matter. Because the rats used here are not substantially larger than adult mice, their vessels are comparably sized. Correspondingly, the results presented here agree well with those seen in adult mice. Moreover, the response of the surface vessels is inversely related to vessel caliber. The smaller vessels of mice might therefore show more constriction than those of adult rats.

At the capillary level, the effect was a dramatic reduction of blood flow. In the normal brain, this transient SD-associated ischemia is too short to cause neuronal death (Nedergaard and Hansen, 1988), but, in the compromised brain, the effects could be more severe. It is therefore significant that the duration of SD-induced capillary flux stop depended on the baseline level of perfusion. The worse the hemodynamic environment of a neuron, the longer the ischemia it experienced during SD. This could explain the susceptibility of the penumbra to each pass of SD wave and its progressive degradation (Back et al., 1996).

SD was also accompanied by $\left[\mathrm{Ca}^{2+}\right]$ waves in neurons and astrocytes. The neuronal $\left[\mathrm{Ca}^{2+}\right]$ wave usually preceded the astrocytic $\left[\mathrm{Ca}^{2+}\right]$ wave but only by a short interval, suggesting that the two waves are tightly coupled. During line-scan experiments, we could not determine the direction of the SD wave. Because the wave can approach from any direction relative to the cells being scanned, on occasion the order of activation of the cells being scanned may reflect only the propagation direction rather than the relative timing of activation of cells simultaneously exposed to an $\mathrm{SD}$ wave. If neither neurons nor astrocytes drive $\mathrm{SD}$, the distribution of lag times should be symmetric at $\sim 0$. If neurons lead but on occasion the wave propagates in a direction from the astrocyte to the neuron, one would expect a minority of cases in which astrocytes appear to be activated first, consistent with our 
findings. This weakens the argument for a driving role for astrocytes in SD propagation, as does the observation that thapsigargin greatly depressed the astrocytic component of the fast wave with no effect on SD propagation. Astrocytes seem to play a passive role in SD propagation, but once recruited, they play an active role in vasoconstriction.

Anoxic depolarizations are SD-like ionic changes that herald irreversible cell damage in ischemia (Hansen, 1985; Martin et al., 1994). The similarity of the $\left[\mathrm{Ca}^{2+}\right]$ waves in $\mathrm{AD}$ to those seen in SD support the similarity of these phenomena and the use of SD as a limited model of $\mathrm{AD}$. Indeed, SD has been shown to occur in the human brain in focal ischemia and trauma (Strong et al., 2002). However, metabolic failure in AD may prevent recovery and leave neurons and astrocytes in a prolonged state of $\mathrm{Ca}^{2+}$ overload, which could trigger excitotoxic events. We show that a drop of cerebral blood flow occurs when SD-associated $\left[\mathrm{Ca}^{2+}\right]$ waves invade astrocytic end feet. Anti-vasoconstrictive treatment may therefore be helpful to optimize residual flow during cerebral ischemia. The contributions of SD-induced vascular versus nonvascular perturbations to the aggravating effect of SD in ischemia have yet to be determined.

\section{References}

Ayata C, Shin HK, Salomone S, Ozdemir-Gursoy Y, Boas DA, Dunn AK, Moskowitz MA (2004) Pronounced hypoperfusion during spreading depression in mouse cortex. J Cereb Blood Flow Metab 24:1172-1182.

Back T, Kohno K, Hossmann KA (1994) Cortical negative DC deflections following middle cerebral artery occlusion and $\mathrm{KCl}$-induced spreading depression: effect on blood flow, tissue oxygenation, and electroencephalogram. J Cereb Blood Flow Metab 14:12-19.

Back T, Ginsberg MD, Dietrich WD, Watson BD (1996) Induction of spreading depression in the ischemic hemisphere following experimental middle cerebral artery occlusion: effect on infarct morphology. J Cereb Blood Flow Metab 16:202-213.

Basarsky TA, Duffy SN, Andrew RD, MacVicar BA (1998) Imaging spreading depression and associated intracellular calcium waves in brain slices. J Neurosci 18:7189-7199.

Bezzi P, Carmignoto G, Pasti L, Vesce S, Rossi D, Rizzini BL, Pozzan T, Volterra A (1998) Prostaglandins stimulate calcium-dependent glutamate release in astrocytes. Nature 391:281-285.

Bures J (1957) The ontogenetic development of steady potential differences in the cerebral cortex in animals. Electroencephalogr Clin Neurophysiol Suppl 9:121-130.

Busch E, Gyngell ML, Eis M, Hoehn-Berlage M, Hossmann KA (1996) Potassium-induced cortical spreading depressions during focal cerebral ischemia in rats: contribution to lesion growth assessed by diffusionweighted NMR and biochemical imaging. J Cereb Blood Flow Metab 16:1090-1099.

Cauli B, Tong XK, Rancillac A, Serluca N, Lambolez B, Rossier J, Hamel E (2004) Cortical GABA interneurons in neurovascular coupling: relays for subcortical vasoactive pathways. J Neurosci 24:8940-8949.

Charles AC, Merrill JE, Dirksen ER, Sanderson MJ (1991) Intercellular signaling in glial cells: calcium waves and oscillations in response to mechanical stimulation and glutamate. Neuron 6:983-992.

Cornell-Bell AH, Finkbeiner SM, Cooper MS, Smith SJ (1990) Glutamate induces calcium waves in cultured astrocytes: long-range glial signaling. Science 247:470-473.

Dani JW, Chernjavsky A, Smith SJ (1992) Neuronal activity triggers calcium waves in hippocampal astrocyte networks. Neuron 8:429-440.

de Luca B, Bures J (1977) Development of cortical spreading depression and of its transition to the caudate nucleus in rats. Dev Psychobiol 10:289-297.

Dirnagl U, Villringer A, Einhaupl KM (1992) In-vivo confocal scanning laser microscopy of the cerebral microcirculation. J Microsc 165:147-157.

Dreier JP, Petzold G, Tille K, Lindauer U, Arnold G, Heinemann U, Einhaupl KM, Dirnagl U (2001) Ischaemia triggered by spreading neuronal activation is inhibited by vasodilators in rats. J Physiol (Lond) 531:515-526.

Dunn AK, Bolay H, Moskowitz MA, Boas DA (2001) Dynamic imaging of cerebral blood flow using laser speckle. J Cereb Blood Flow Metab 21:195-201.

Fabricius M, Akgoren N, Lauritzen M (1995) Arginine-nitric oxide pathway and cerebrovascular regulation in cortical spreading depression. Am J Physiol 269:H23-H29.

Fabricius M, Akgoren N, Dirnagl U, Lauritzen M (1997) Laminar analysis of cerebral blood flow in cortex of rats by laser-Doppler flowmetry: a pilot study. J Cereb Blood Flow Metab 17:1326-1336.

Fabricius M, Fuhr S, Bhatia R, Boutelle M, Hashemi P, Strong AJ, Lauritzen M (2006) Cortical spreading depression and peri-infarct depolarization in acutely injured human cerebral cortex. Brain 129:778-790.

Faraci FM, Sobey CG (1998) Role of potassium channels in regulation of cerebral vascular tone. J Cereb Blood Flow Metab 18:1047-1063.

Filosa JA, Bonev AD, Nelson MT (2004) Calcium dynamics in cortical astrocytes and arterioles during neurovascular coupling. Circ Res 95:e73-e81.

Finkbeiner S (1992) Calcium waves in astrocytes-filling in the gaps. Neuron 8:1101-1108.

Flemming R, Cheong A, Dedman AM, Beech DJ (2002) Discrete storeoperated calcium influx into an intracellular compartment in rabbit arteriolar smooth muscle. J Physiol (Lond) 543:455-464.

Giaume C, Venance L (1998) Intercellular calcium signaling and gap junctional communication in astrocytes. Glia 24:50-64.

Goadsby PJ (1992) The oligemic phase of cortical spreading depression is not blocked by tirilazad mesylate (U-74006F). Brain Res 588:140-143.

Gold L, Back T, Arnold G, Dreier J, Einhaupl KM, Reuter U, Dirnagl U (1998) Cortical spreading depression-associated hyperemia in rats: involvement of serotonin. Brain Res 783:188-193.

Hansen AJ (1985) Effect of anoxia on ion distribution in the brain. Physiol Rev 65:101-148.

Haydon PG, Carmignoto G (2006) Astrocyte control of synaptic transmission and neurovascular coupling. Physiol Rev 86:1009-1031.

Hirase H, Qian L, Bartho P, Buzsaki G (2004) Calcium dynamics of cortical astrocytic networks in vivo. PLoS Biol 2:E96.

Iadecola C (2004) Neurovascular regulation in the normal brain and in Alzheimer's disease. Nat Rev Neurosci 5:347-360.

James MF, Smith JM, Boniface SJ, Huang CL, Leslie RA (2001) Cortical spreading depression and migraine: new insights from imaging? Trends Neurosci 24:266-271.

Jarvis CR, Anderson TR, Andrew RD (2001) Anoxic depolarization mediates acute damage independent of glutamate in neocortical brain slices. Cereb Cortex 11:249-259.

Kleinfeld D, Mitra PP, Helmchen F, Denk W (1998) Fluctuations and stimulus-induced changes in blood flow observed in individual capillaries in layers 2 through 4 of rat neocortex. Proc Natl Acad Sci USA 95:15741-15746.

Kunkler PE, Kraig RP (1998) Calcium waves precede electrophysiological changes of spreading depression in hippocampal organ cultures. J Neurosci 18:3416-3425.

Lacombe P, Sercombe R, Correze JL, Springhetti V, Seylaz J (1992) Spreading depression induces prolonged reduction of cortical blood flow reactivity in the rat. Exp Neurol 117:278-286.

Lauritzen M, Fabricius M (1995) Real time laser-Doppler perfusion imaging of cortical spreading depression in rat neocortex. NeuroReport 6:1271-1273

Leão AAP (1944) Spreading depression of activity in the cerebral cortex. J Neurophysiol 7:357-390.

Martin RL, Lloyd HG, Cowan AI (1994) The early events of oxygen and glucose deprivation: setting the scene for neuronal death? Trends Neurosci 17:251-257.

Martins-Ferreira H, Nedergaard M, Nicholson C (2000) Perspectives on spreading depression. Brain Res Brain Res Rev 32:215-234.

Metea MR, Newman EA (2006) Glial cells dilate and constrict blood vessels: a mechanism of neurovascular coupling. J Neurosci 26:2862-2870.

Mulligan SJ, MacVicar BA (2004) Calcium transients in astrocyte endfeet cause cerebrovascular constrictions. Nature 431:195-199.

Nedergaard M (1996) Spreading depression as a contributor to ischemic brain damage. Adv Neurol 71:75-83; discussion 83-84.

Nedergaard M, Hansen AJ (1988) Spreading depression is not associated with neuronal injury in the normal brain. Brain Res 449:395-398.

Nielsen AN, Fabricius M, Lauritzen M (2000) Scanning laser-Doppler flow- 
metry of rat cerebral circulation during cortical spreading depression. J Vasc Res 37:513-522.

Nimmerjahn A, Kirchhoff F, Kerr JN, Helmchen F (2004) Sulforhodamine 101 as a specific marker of astroglia in the neocortex in vivo. Nat Methods 1:31-37.

Pasti L, Zonta M, Pozzan T, Vicini S, Carmignoto G (2001) Cytosolic calcium oscillations in astrocytes may regulate exocytotic release of glutamate. J Neurosci 21:477-484.

Peters A, Palay SL, Webster HD (1991) Blood vessels. In: The fine structure of the nervous system, Ed 3, pp 344-355. New York: Oxford UP.

Peters O, Schipke CG, Hashimoto Y, Kettenmann H (2003) Different mechanisms promote astrocyte $\mathrm{Ca}^{2+}$ waves and spreading depression in the mouse neocortex. J Neurosci 23:9888-9896.

Seitz I, Dirnagl U, Lindauer U (2004) Impaired vascular reactivity of isolated rat middle cerebral artery after cortical spreading depression in vivo. J Cereb Blood Flow Metab 24:526-530.

Shin HK, Dunn AK, Jones PB, Boas DA, Moskowitz MA, Ayata C (2006) Vasoconstrictive neurovascular coupling during focal ischemic depolarizations. J Cereb Blood Flow Metab 26:1018-1030.

Somjen GG (2001) Mechanisms of spreading depression and hypoxic spreading depression-like depolarization. Physiol Rev 81:1065-1096.

Stosiek C, Garaschuk O, Holthoff K, Konnerth A (2003) In vivo two-photon calcium imaging of neuronal networks. Proc Natl Acad Sci USA 100:7319-7324.

Street IP, Lin HK, Laliberte F, Ghomashchi F, Wang Z, Perrier H, Tremblay NM, Huang Z, Weech PK, Gelb MH (1993) Slow- and tight-binding inhibitors of the $85-\mathrm{kDa}$ human phospholipase A2. Biochemistry 32:5935-5940.

Strong AJ, Fabricius M, Boutelle MG, Hibbins SJ, Hopwood SE, Jones R, Parkin MC, Lauritzen M (2002) Spreading and synchronous depressions of cortical activity in acutely injured human brain. Stroke 33:2738-2743.

Takano T, Tian GF, Peng W, Lou N, Libionka W, Han X, Nedergaard M (2005) Astrocyte-mediated control of cerebral blood flow. Nat Neurosci 9:260-267.

Tomita M, Schiszler I, Tomita Y, Tanahashi N, Takeda H, Osada T, Suzuki N (2005) Initial oligemia with capillary flow stop followed by hyperemia during $\mathrm{K}^{+}$-induced cortical spreading depression in rats. J Cereb Blood Flow Metab 25:742-747.

Wang Z, Tymianski M, Jones OT, Nedergaard M (1997) Impact of cytoplasmic calcium buffering on the spatial and temporal characteristics of intercellular calcium signals in astrocytes. J Neurosci 17:7359-7371.

Zonta M, Angulo MC, Gobbo S, Rosengarten B, Hossmann KA, Pozzan T, Carmignoto G (2003) Neuron-to-astrocyte signaling is central to the dynamic control of brain microcirculation. Nat Neurosci 6:43-50. 